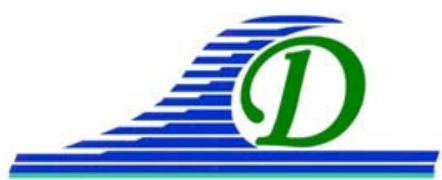
XIII ${ }^{\text {èmes }}$ Journées Nationales Génie Côtier - Génie Civil
Dunkerque, 2-4 juillet 2014

DOI:10.5150/jngcgc.2014.071 @ Editions Paralia CFL

disponible en ligne - $h$ ttp://www.paralia.fr - available online

\title{
Conception d'un atténuateur de houle de type caisson à double parois poreuses
}

\section{David LAJOIE ${ }^{1}$, Jean BOUGIS ${ }^{2}$, Julien DOLIDON ${ }^{3}$}

1. Ingénieur Conseil, 06323656 92, 06530 Le Tignet, France.

david.lajoie@hydrogc.fr

2. Ingénieur Conseil, 060730 70 38, 06650 Opio, France.

jean.bougis@wanadoo.fr

3. Maître d’ouvrage CCI Nice Côte d’Azur, 06300 Nice, France. 0492004353

julien.dolidon@cote-azur.cci.fr

\section{Résumé :}

La rénovation du vieux port de Cannes a incité la CCI Nice Côte d'Azur à tenter d'améliorer le confort du port, altéré depuis la construction en 1995 d'un mur de parking réfléchissant la houle au fond du bassin. Le besoin de restructurer entièrement le ponton Pantiero, implanté au milieu du bassin, était aussi l'occasion d'apporter de la dissipation supplémentaire à travers la conception d'un nouvel ouvrage. Parmi les solutions appropriées, le caisson Jarlan présentait des avantages indéniables. Les études hydrodynamiques classiques en agitation portuaire ont mis en évidence l'intérêt de concevoir un atténuateur double flux, se comportant comme un caisson Jarlan en termes de réflexion des houles incidentes dominantes, mais autorisant une certaine transmission d'énergie. Cela se traduit par le remplacement de la paroi habituellement opaque en fond de Jarlan par une paroi faiblement poreuse, laquelle évite les réflexions totales à l'aval de l'ouvrage et limite les résonances par réflexions entre deux parois verticales dans un port. Des simulations en interaction houle-structure intégrant les effets de pertes de charge à travers des plaques poreuses ont permis de cerner l'ouvrage en phase de conception. Les paramètres de dimensionnement ont ensuite été validés en canal à houle dans le cadre des études d'exécution de l'ouvrage construit en 2013.

Mots-clés: Agitation portuaire, Atténuateurs de houle, Caissons Jarlan, Parois poreuses.

\section{Introduction}

La construction du parking Pantiero au fond du vieux port de Cannes dans les années 1990 s'est accompagnée de la mise en œuvre sur une ancienne plage d'une paroi verticale réfléchissant les vagues résiduelles, engendrant logiquement une dégradation de l'agitation du plan d'eau.

La CCI Nice Côte d'Azur, en tant qu'exploitant du port, a souhaité coupler ses projets de restructuration de l'aménagement intérieur du port avec une réflexion sur les possibilités d'améliorer la protection du plan d'eau. L'une des pistes les plus judicieuses 


\section{Thème 4 - Ouvrages portuaires et offshore}

consistait à profiter de la reconstruction du vieux ponton Pantiero, structure fixe en forme de $T$, constituée à l'origine d'un tablier en béton armé posé sur pieux, sur lequel sont arrimés des pontons flottants classiques, formant le peigne Pantiero, accueillant les navires de plaisance. La nouvelle structure pouvait offrir des propriétés dissipatrices, en évitant, a priori, de modifier les limites physiques existantes du port. Il importait par ailleurs de conserver approximativement l'emprise actuelle de l'ouvrage, au moins sur les paramètres longueur et largeur, de manière à conserver l'implantation des mouillages, même si celui-ci pouvait être éventuellement déplacé à l'intérieur du bassin. Parmi les ouvrages atténuateurs couramment utilisées, le caisson Jarlan s'imposait comme l'une des solutions les plus appropriées pour reconstituer une nouvelle infrastructure maritime du ponton Pantiero. Les diverses modélisations théoriques, d'abord en agitation portuaire, puis en interaction houle-structure des effets atténuateurs des caissons, ont ouvert de nouvelles pistes sur une structure de caisson variante, consistant à rendre poreuse la paroi aval, laquelle est en principe opaque sur les caissons Jarlan classiques.

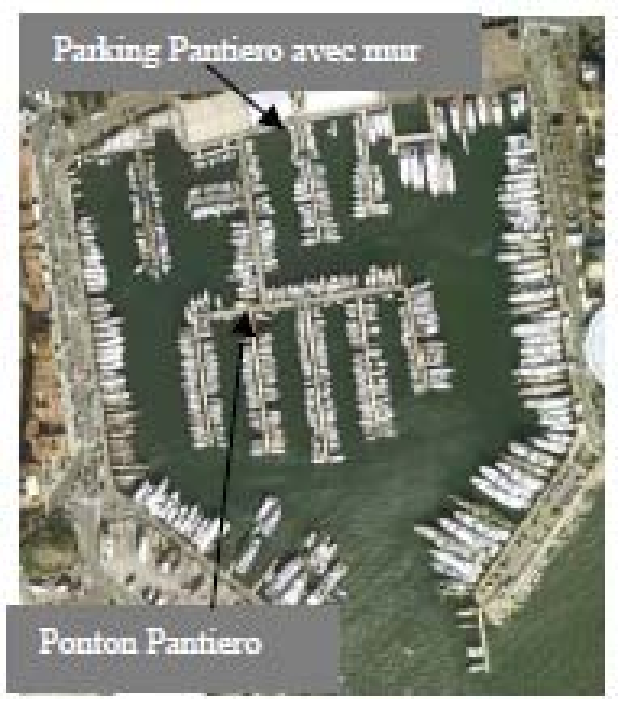

Figure 1. Vieux Port de Cannes.

On développe ci-après les principes théoriques qui ont conduit à concevoir une telle structure au milieu d'un bassin portuaire. Les résultats espérés ont été confirmés par des essais en canal à houle, pour lesquels plusieurs précautions ont été prises de manière à représenter convenablement les effets de pertes de charge à travers les diverses porosités.

\section{L'agitation du bassin portuaire}

L'agitation dans le bassin portuaire a été modélisée de façon classique par résolution de l'équation de diffraction-réfraction combinée de Berkhoff par une méthode aux 


\section{XIII ${ }^{\text {èmes }}$ Journées Nationales Génie Côtier - Génie Civil \\ Dunkerque, 2-4 juillet 2014}

éléments finis (bibliothèque Coelacanthe d'hydratec, développements communs $\mathrm{J}$. BOUGIS - D. LAJOIE). Les principes de modélisation sont les suivants :

- frontière ouverte circulaire, où l'on applique les conditions de houle incidentes via le potentiel complexe $\varphi_{I}$ et sa vitesse normale $\partial \varphi_{I} / \partial n$, ainsi que les conditions de rayonnement des ondes réfléchies vers le large ;

- frontières physiques classiques où l'on applique des coefficients de réflexion partielle traduisant les effets amortisseurs des digues à talus ( $K r=0.4-0.5$ selon la cambrure) ou les réflexions quasi-totales des parois verticales ;

- frontières physiques particulières, où l'on exprime des coefficients de réflexion partiels variables selon la période de houle (cas des caissons Jarlan par exemple) ou des conditions de réflexion-transmission. Celles-ci peuvent être de type superélément reliant l'ensemble des potentiels $\varphi$ de tous les points de la frontière à l'ensemble des vitesses normales $\partial \varphi / \partial n$ par des conditions matricielles établies préalablement à l'aide d'un modèle houle-structure 2D ou 3D (LAJOIE, 1996), (BOUGIS \& DEGAIE 1996). En phase de recherche des valeurs appropriées des coefficients de réflexion et de transmission, ou lorsque l'ouvrage est élancé (longueur $>>$ largeur), il est judicieux d'appliquer une condition aux limites traduisant la réflexion et la transmission partielle à travers un élément de type "barre" en tenant compte d'un fonctionnement dissymétrique, avec réflexions $r_{A}, r_{B}$ côtés $\mathrm{A}$ ou B de l'ouvrage et transmissions $t_{A \rightarrow B}, t_{B \rightarrow A}$ :

$\frac{\partial \varphi_{A}}{\partial n}=i k_{A} \frac{\left[\left(1-r_{A}\right)\left(1+r_{B}\right) \sin \theta_{A}+t_{A \rightarrow B} t_{B \rightarrow A} \sin \theta_{B}\right] \varphi_{A}-t_{B \rightarrow A}\left[\left(1-r_{A}\right) \sin \theta_{A}+\left(1+r_{B}\right) \sin \theta_{B}\right] \varphi_{B}}{\left(1+r_{A}\right)\left(1+r_{B}\right)-t_{A \rightarrow B} t_{B \rightarrow A}}$

Coté $B$

La condition aux limites ci-dessus permet de relier 2 à 2 les points se faisant face de part et d'autre l'ouvrage. L'approche est bidimensionnelle horizontale, c'est-à-dire que les effets de bord par diffraction aux extrémités de l'ouvrage ne sont pas correctement pris en compte. L'erreur est d'autant plus réduite que l'ouvrage est élancé, ce qui est le cas du peigne Pantiero ; 


\section{Thème 4 - Ouvrages portuaires et offshore}

- analyse spectrale à partir des nombreux champs d'agitation issus des balayages systématiques en périodes et directions de manière à établir les champs de hauteurs $H_{S}$ des houles irrégulières multidirectionnelles.

Les simulations des champs d'agitation, d'abord dans la configuration initiale du port, puis en introduisant divers couples $(K r, K t)$ réalistes à travers l'ouvrage ont apporté les enseignements suivants :

- L'application de conditions aux limites classiques ( $K r=0.5$ sur les talus en enrochements, $K r=0.95$ sur les parois verticales) a permis de reproduire la réalité physique observé, à savoir un port fortement agité dans le fond du bassin. Des mesures ponctuelles d'agitation sur 3 points caractéristiques, menées par Nortek Med lors d'un coup de mer soutenu mais fréquent, sont venus valider a posterori les valeurs de hauteurs significatives $H s$ calculées dans la configuration initiale du port.

- Un simple caisson Jarlan à l'emplacement du ponton Pantiero, avec des ouvertures côté large offrant un coefficient de réflexion $K r$ de l'ordre de 0.4, apporte une accalmie globale du bassin grâce aux effets de dissipation supplémentaire par rapport à la situation initiale. Logiquement, des modes résonnants s'installent néanmoins entre le mur du parking Pantiero au fond du bassin et la paroi opaque à l'arrière du caisson. Il s'ensuit quelques altérations locales du niveau d'agitation vers le fond du bassin.

- L'application d'une réflexion partielle à l'arrière du caisson Jarlan permet de réduire les effets des modes résonants. Une dissipation d'énergie sur la face arrière d'un caisson Jarlan peut être obtenue en rendant poreuse la paroi initialement opaque. Disposant de peu d'espace sur la largeur de l'ouvrage, environ $5 \mathrm{~m}$ ce qui est déjà dans les limites acceptables pour qu'un caisson Jarlan soit efficace face aux houles fréquemment gênantes de périodes comprises entre $5 \mathrm{~s}$ et $8 \mathrm{~s}$, il n'est pas approprié d'accoler 2 caissons Jarlan dos à dos, avec une paroi opaque entre deux parois poreuses. Dans ces conditions, on se limite à deux parois espacées de $5 \mathrm{~m}$, si bien que la perforation de la paroi opaque à l'arrière du caisson s'accompagne d'une certaine transmission d'énergie. L'approche par tâtonnement numérique sur les champs d'agitation montre que le meilleur compromis est obtenu en imposant un coefficient de réflexion $\mathrm{Kr} \approx 0.6$ à 0.7 , accompagné d'un coefficient de transmission n'excédant pas $K t \approx 0.3$ à 0.4 . 


\section{XIII ${ }^{\text {èmes }}$ Journées Nationales Génie Côtier - Génie Civil \\ Dunkerque, 2-4 juillet 2014}

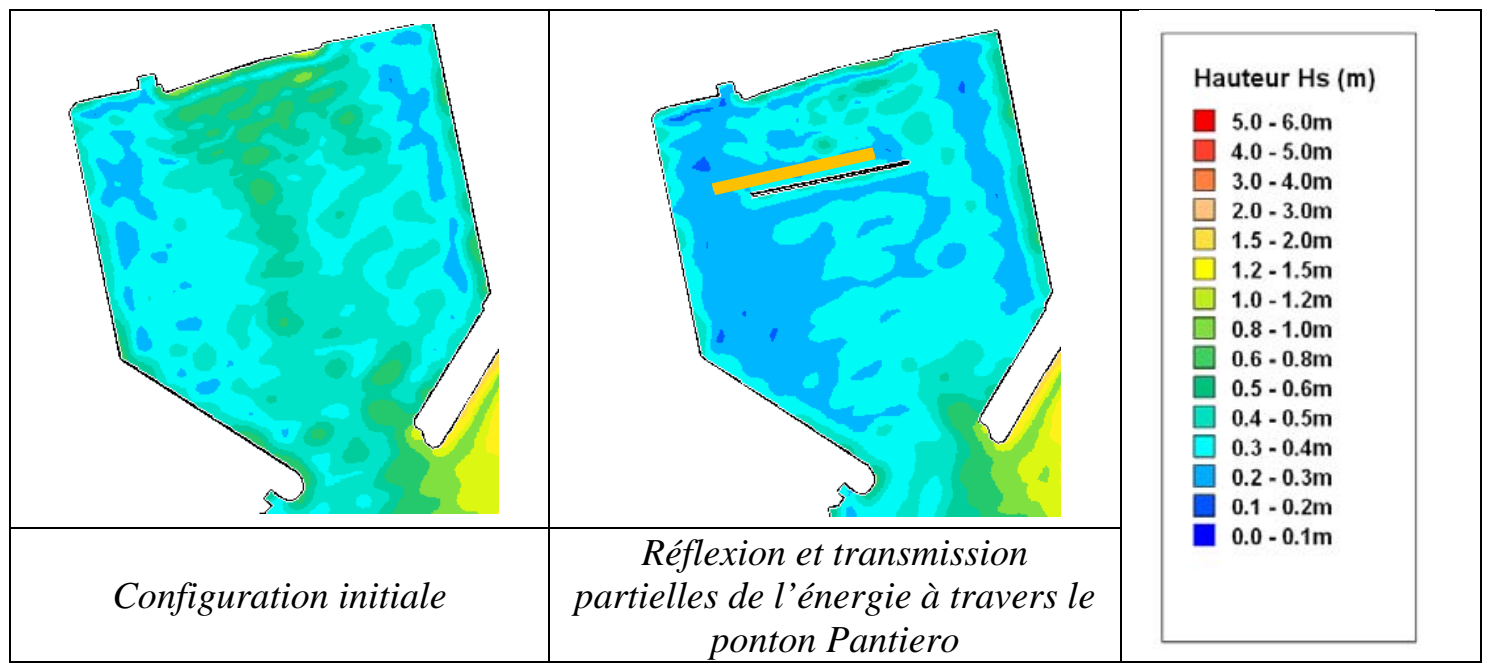

Figure 2. Champs d'agitation en occurrence décennale dans le vieux port de Cannes.

\section{Modèle d'interaction houle-parois poreuse}

Le problème d'interaction houle-structure est résolu par une approche classique de résolution du problème de diffraction $2 \mathrm{D}$ vertical par une méthode de singularités, en admettant la théorie potentielle des ondes linéaires en dehors des zones de dissipation visqueuse engendrée par les parois poreuses.

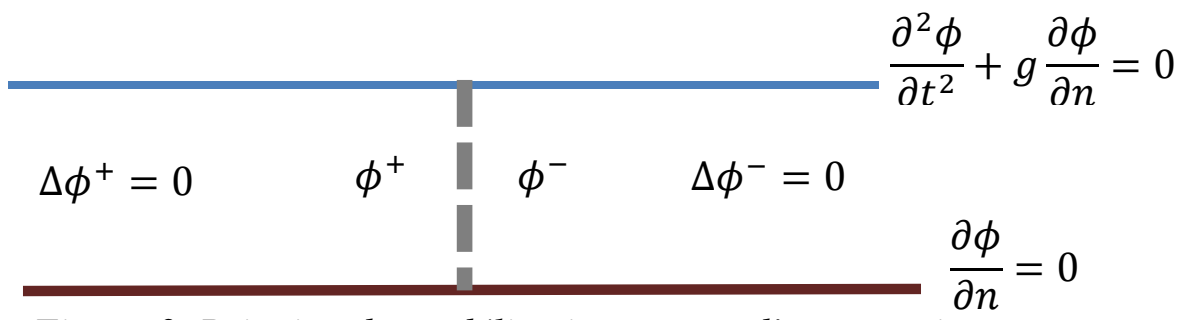

Figure 3. Principe de modélisation autour d'une paroi poreuse.

Les pertes de charge à travers les perforations s'expriment de la façon suivante après intégration de l'équation de Lagrange le long d'une ligne de courant traversant une perforation sur une longueur 1 :

$P^{+}-P^{-}=\frac{1}{2} \rho K|V| V+\int_{0}^{l} \rho \frac{1}{\varepsilon k_{c}} \frac{\partial V}{\partial t} \cdot d l$

$V$ est la vitesse moyenne de l'écoulement à l'amont ou à l'aval des perforations ;

$\varepsilon$ est le taux de porosité ;

$k_{c}$ est le coefficient de striction de section lors du passage d'un jet dans une ouverture ; $K$ est le coefficient de pertes de charge à travers la plaque poreuse, dont une expression appropriée est :

$\frac{1-\varepsilon}{\varepsilon^{2} k_{c}^{2}}$

En retenant l'hypothèse des ondes monochromatiques, et après linéarisation de Lorentz des termes quadratiques, l'expression ci-dessus se traduit par l'équation suivante reliant 


\section{Thème 4 - Ouvrages portuaires et offshore}

le potentiel complexe des vitesses $\phi^{+}$et $\phi^{-}$de part et d'autre de la paroi poreuse, à la vitesse normale moyenne à travers la paroi (LAJOIE, 2008) :

$\frac{\partial \phi}{\partial n}=\frac{\phi^{+}-\phi^{-}}{-i \frac{4 K}{3 \pi \omega}\left\{\frac{l^{2}}{\varepsilon^{2} k_{c}^{2}}+\sqrt{\frac{l^{4}}{\varepsilon^{4} k_{C}^{4}}+4\left(\frac{4 K}{3 \pi \omega}\left\|\phi^{+}-\phi^{-}\right\|\right)^{2}} / 2\left(\frac{4 K}{3 \pi \omega}\right)^{2}\right\}-\frac{l}{\varepsilon k_{c}}}$

L'approche numérique est comparée à des résultats d'essais en laboratoire sur une houle traversant une simple paroi poreuse (ALLSOP \& HETTIARACHCHI, 1988). Les coefficients de réflexion $K_{r}$ et de transmission $K_{t}$ ont été mesurés en canal à houle pour plusieurs taux de porosité allant de $5 \%$ à $25 \%$ et pour plusieurs valeurs du rapport profondeur / longueur d'onde à la période pic $h / \lambda_{P}$. Sur les graphes ci-dessous, on compare les valeurs obtenues dans une configuration généralement rencontrée dans les ports, dont celui de Cannes : houle longue $h / \lambda_{P}=0.1$, soit $\mathrm{T}_{\mathrm{P}}=7.5 \mathrm{~s}, \lambda_{\mathrm{P}}=50 \mathrm{~m}$ pour $h=5 \mathrm{~m}$, faible cambrure $\approx 1 \%$. Les allures générales des courbes $K_{r}$ et $K_{t}$ en fonction du taux de porosité $\tau$ sont correctement reproduites par l'approche numérique. Les valeurs absolues pour les hauteurs de houle incidente $H=0.3 \mathrm{~m}$ et $H=0.5 \mathrm{~m}$ encadrent les valeurs mesurées en adoptant une longueur caractéristique de l'écoulement à travers les perforations de l'ordre de $H / 2$ et un coefficient de striction de section $k_{C}=0.8$. Cette valeur de $k_{C}$, qui est associée à un écoulement oscillant, est logiquement supérieure à la valeur théorique $k_{C}=0.65$ qui serait applicable à un écoulement permanent établi.

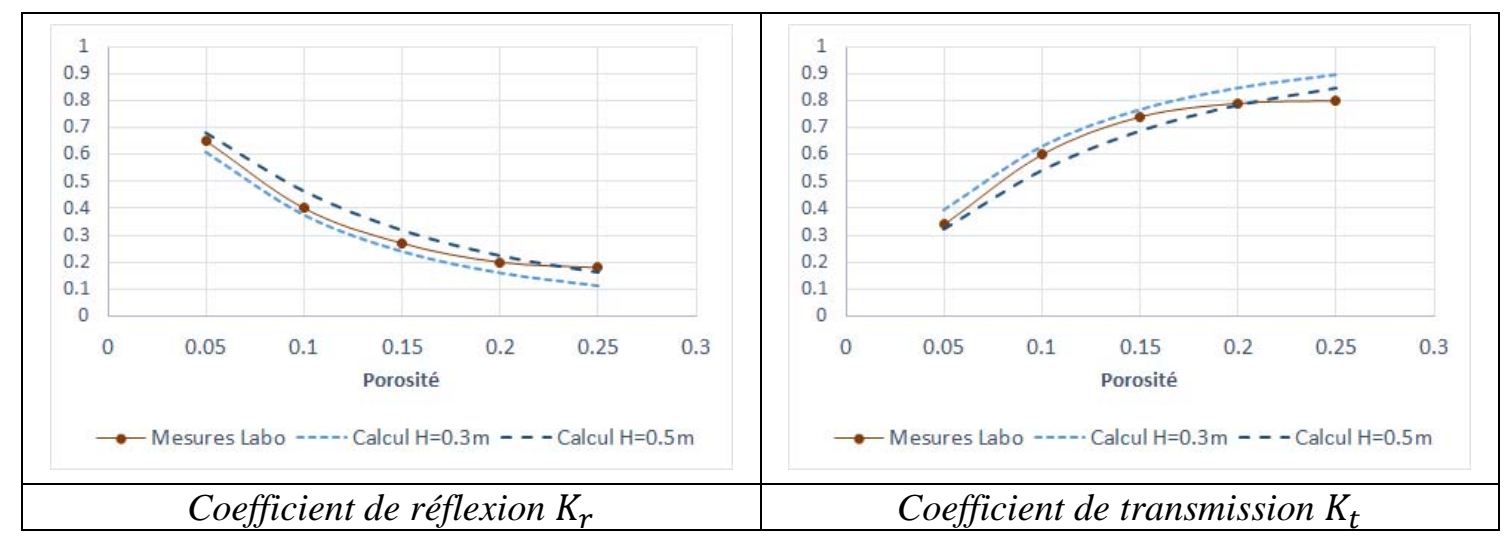

Figure 4. Comparaison mesures-calcul réfléxion transmission à travers une paroi poreuse simple.

Dans cette configuration d'ondes longues, le taux de réflexion $K r$ dépend essentiellement de la porosité, un peu de la hauteur incidente $H$, et très peu de la période. Pour l'ensemble des simulations, on retrouve sensiblement l'égalité $K_{r}+K_{t} \approx$ 1 , ce que l'on constate également pour toutes les configurations testées en canal à houle. Le taux de dissipation d'énergie, égal à $1-K_{r}^{2}-K_{t}^{2}$, atteint un maximum de $50 \%$ lorsque $K_{r}=K_{t}=0.5$ et vaut encore plus de $40 \%$ pour les couples $\left[K_{r} ; K_{t}\right]=$ $[0.6 ; 0.4]$ à $[0.7 ; 0.3]$. 


\section{XIII ${ }^{\text {èmes }}$ Journées Nationales Génie Côtier - Génie Civil \\ Dunkerque, 2-4 juillet 2014}

Le caisson à double parois poreuses doit être conçu pour se comporter comme un caisson Jarlan côté amont, celui ouvert vers le large, et comme une simple paroi poreuse côté aval, faisant face au mur droit du parking Pantiero. Dans le sens large $\rightarrow$ mur, il importe de minimiser les réflexions en maîtrisant les transmissions $\left(K_{t}<0.3\right.$ à 0.4$)$, tandis que dans le sens mur $\rightarrow$ large, il suffit de casser les résonances avec un coefficient de réflexion modéré $\left(K_{r} \approx 0.6\right.$ à 0.7$)$

Ce fonctionnement est atteint en imposant une porosité relativement grande sur la paroi amont, $\varepsilon_{1}=15 \%$, généralement applicable aux caissons Jarlan, et une porosité faible sur la paroi aval $\varepsilon_{2}=5 \%$. Cette dissymétrie de porosité permet de conserver les propriétés de faible réflexion à l'amont, inhérente au fonctionnement des caissons Jarlan. Les résultats de simulation en termes de coefficients $K_{r}$ et $K_{t}$ sont présentés ciaprès en même temps que leur comparaison avec les mesures en canal à houle.

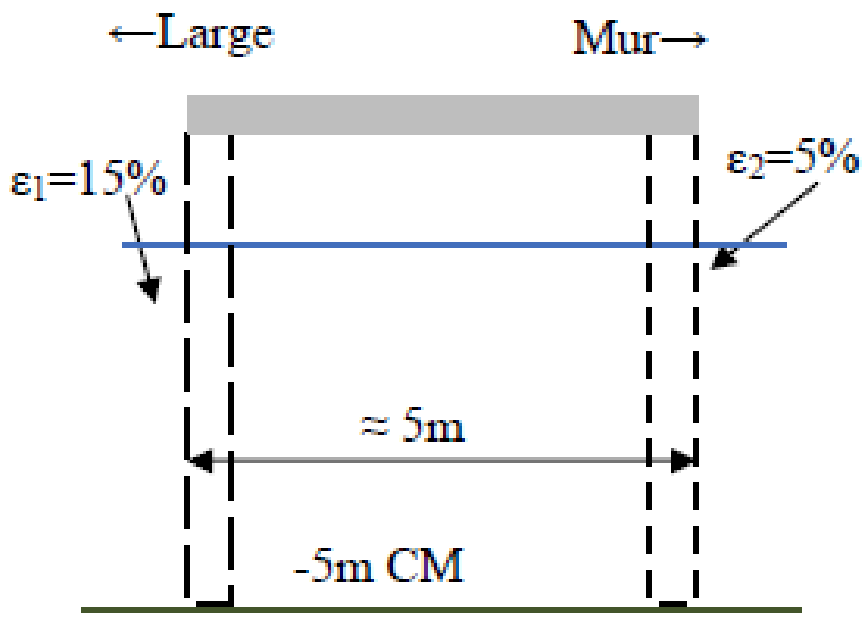

Figure 5. Principe de la double paroi poreuse.

\section{Essais en canal à houle}

Le modèle numérique fait intervenir des paramètres physiques, comme le coefficient de striction et le coefficient de pertes de charge de l'écoulement au passage des perforations, qui sont délicats à évaluer théoriquement. Or ils jouent un rôle fondamental dans les variations des coefficients de réflexion et de transmission de la houle, mais aussi des efforts hydrodynamiques, en fonction de l'écartement des parois et de leurs porosités. Il est donc apparu nécessaire de réaliser une campagne d'essais sur modèle physique en canal à houle pour affiner ces paramètres et fiabiliser les résultats.

Désignons par $U$ la vitesse à travers la perforation de diamètre hydraulique $D$, par $E$ l'épaisseur de la paroi perforée et par $v$ la viscosité cinématique du fluide.

L'analyse dimensionnelle montre que quatre similitudes importantes interviennent dans ce type de modèle : 


\section{Thème 4 - Ouvrages portuaires et offshore}

- la similitude de Froude qui doit être totalement respectée pour que la modélisation de la houle ait un sens ;

- la similitude de porosité qui impose de conserver les porosités des parois ;

- la similitude de Reynolds des performations $(\operatorname{Re}=U D / v)$ qui ne doit pas être trop violemment distordue pour que le régime d'écoulement à travers les parois poreuses soit conservé sur le modèle ;

- la similitude de pertes de charge dans les perforations qui exige la conservation du rapport $E / D$ entre l'épaisseur des canaux perforés et leur diamètre hydraulique.
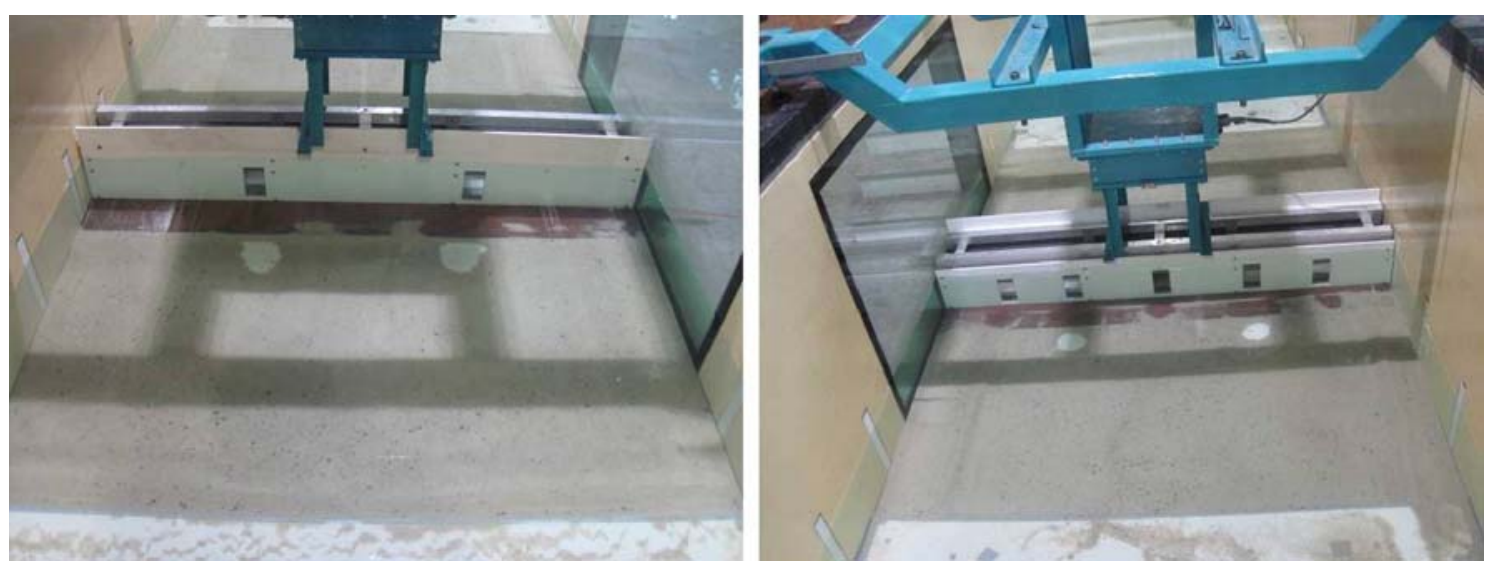

Figure 6. Paroi poreuses 5\% à gauche et $15 \%$ à droite

(canal à houle d'ACRI $I_{I N}$, Sophia Antipolis).

Il en résulte d'abord que l'échelle doit être la plus grande possible, compatible avec la capacité du batteur de l'installation (hauteur et période de la houle) et avec celle de l'instrumentation (mesure de faibles hauteurs de houle. L'échelle du $1 / 20^{\mathrm{e}}$ s'est révélée être le meilleur compromis possible entre toutes les exigences.

Les parois poreuses du prototype étant réalisées en tôles perforées d'une dizaine de mm d'épaisseur, il était impossible de les modéliser à l'échelle en leur conservant une rigidité suffisante. L’agrandissement des perforations à porosité constante s'est avéré nécessaire pour conserver le rapport $E / D$ (voir figure 6). Heureusement, ce regroupement conduit également à diminuer sensiblement la distorsion du nombre de Reynolds.

Pour obtenir les coefficients de réflexion et de transmission dans chaque sens de propagation de la houle, les essais ont été réalisés deux fois en retournant la maquette.

La figure 7 présente les résultats des essais et des calculs réalisés. 


\section{XIII ${ }^{\text {èmes }}$ Journées Nationales Génie Côtier - Génie Civil \\ Dunkerque, 2-4 juillet 2014}

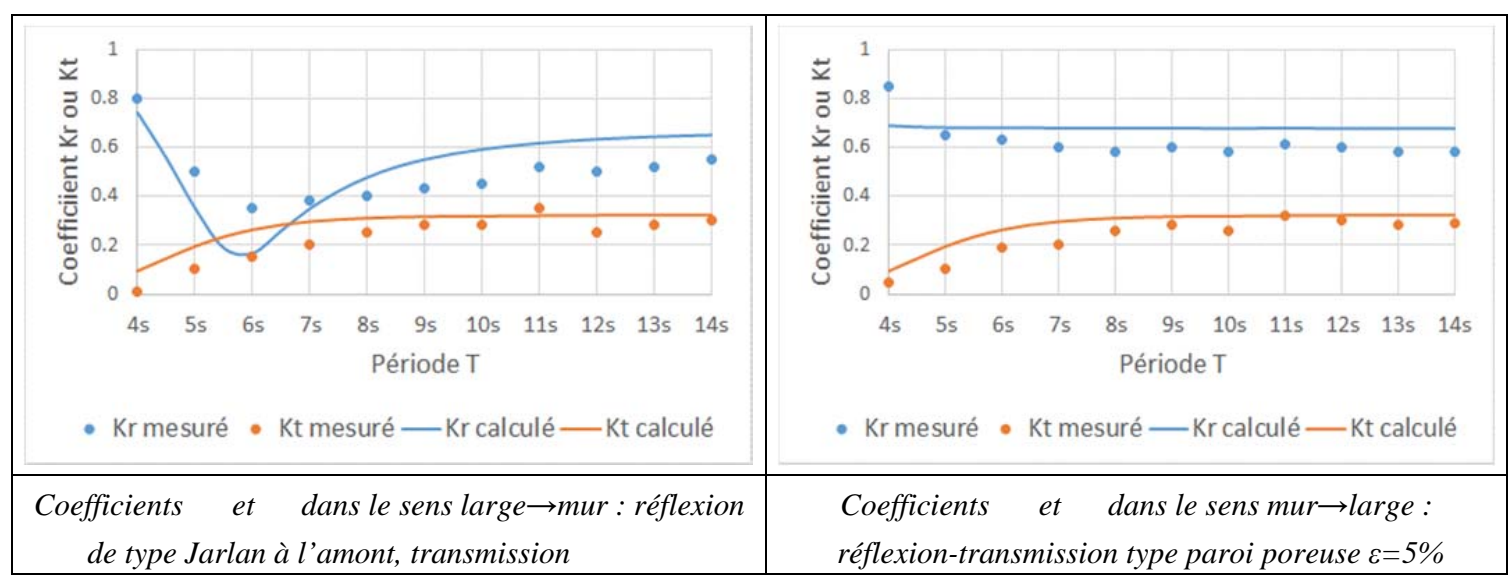

Figure 7. Comparaison mesures-calculs réflexion-transmission à travers un caisson double parois poreuses.

Le calcul numérique permet de prédire les bonnes tendances en termes de coefficients de réflexion et de transmission. On notera que le calcul numérique est trop optimiste autour du pic optimal de fonctionnement autour de $T=5 \mathrm{~s}$ à $7 \mathrm{~s}$, indiquant une valeur du coefficient de réflexion $K r$ inférieure à ce qui a été mesuré en canal à $T=6 \mathrm{~s}$. Ceci pourrait s'expliquer par la non prise en compte au niveau du modèle théorique du fonctionnement non linéaire de l'ouvrage. Un transfert d'énergie vers les hautes fréquences, particulièrement au voisinage de la résonance (aux faibles valeurs de $K r$ ), pourrait être à l’origine de cet écart.

\section{Conclusion}

La configuration du Vieux Port de Cannes incitait à exploiter le besoin de rénovation complète de la structure du ponton Pantiero pour dissiper efficacement l'énergie de façon à compenser en partie les effets de réflexion induits par le mur du parking au fond du bassin. Parmi les solutions connues, le caisson Jarlan apparaissait comme une solution appropriée. La simulation numérique à la fois en agitation portuaire et en interaction houle-structure ont ouvert une voie pour une solution de type double parois poreuses, a priori plus optimisée dans cette configuration particulière. Les essais en canal à houle ont confirmé le fonctionnement attendu dans les deux sens. L'ouvrage a été construit en 2013 par l'Entreprise Bouygues TPRF sous maîtrise d'œuvre Arcadis ESG.

\section{Références bibliographiques}

ALLSOP N.W., HETTIARACHCHI S.S. (1988). Reflections from Coastal Structures. $21^{\text {st }}$ International Coastal Engineering Conference, ASCE, Vol. 1, pp 782-794. 
Thème 4-Ouvrages portuaires et offshore

BOUGIS J., DEGAIE A. (1996). Modélisation numérique du comportement d'atténuateurs de houle flottants sur un site côtier. IV ${ }^{\text {èmes }}$ Journées Nationales Génie Côtier - Génie Civil, Dinard, pp 381-388. http://dx.doi.org/10.5150/jngcgc.1996.038-B

LAJOIE D. (1996). Modélisation de la houle en zone côtière : prévision de l'agitation à l'intérieur des ports et mise au point d'atténuateurs de houle dynamiques. Thèse de l’Université Aix-Marseille II.

LAJOIE D. (2008). Optimisation du fonctionnement des atténuateurs de houle de type "dos de chameau" à l'aide de perforations dans la structure. $\mathrm{X}^{\mathrm{èmes}}$ Journées Nationales Génie Côtier - Génie Civil, Sophia Antipolis, pp 749-759. http://dx.doi.org/10.5150/ingcgc.2008.071-L 